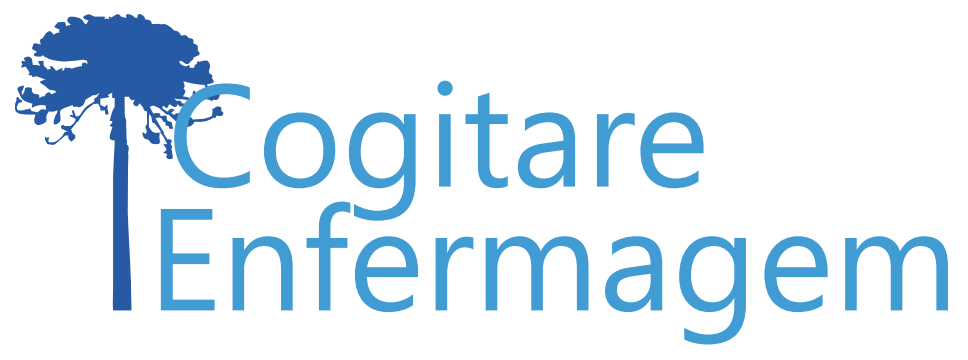

\title{
EXPRESSÃO DAS TENDÊNCIAS PEDAGÓGICAS EM TESES E DISSERTAÇÕES DE UM LABORATÓRIO DE PESQUISA E TECNOLOGIA EM EDUCAÇÃO EM ENFERMAGEM E SAÚDE (1994-2017)
}

André Lucas Maffissoni ${ }^{1}$, Marina da Silva Sanes², Franciane Schneider ${ }^{3}$, Jussara Gue Martini ${ }^{4}$, Monica Motta Lino ${ }^{5}$, Silvana Silveira Kempfer ${ }^{6}$

\section{RESUMO}

Objetivo: analisar como as tendências pedagógicas se expressam nas investigações científicas do Laboratório de Pesquisa e Tecnologia em Educação em Enfermagem e Saúde, da Universidade Federal de Santa Catarina.

Método: pesquisa qualitativa, utilizando análise documental de teses e dissertações produzidas no período 1994-2017. A coleta dos dados ocorreu de março a maio de 2018. Dados tratados à luz da análise de conteúdo.

Resultados: identificaram-se 106 documentos. Destes, 89 se filiam à tendência libertadora/criativa, 17 se aproximam da tendência progressista e não foram evidenciados estudos exclusivamente relacionados à tendência pedagógica convencional.

Conclusões: as teses e dissertações do Laboratório de Pesquisa trabalharam a educação em enfermagem a partir de perspectiva pautada, majoritariamente, pela tendência pedagógica libertadora/criativa. Este estudo contribui para a construção do conhecimento na enfermagem por reconhecer os pressupostos pedagógicos utilizados por pesquisadores da área, essencial para identificar quais posicionamentos políticos a enfermagem brasileira assume em suas investigações científicas.

DESCRITORES: Pesquisa em enfermagem; Grupos de pesquisa; Modelos educacionais; Educação em enfermagem; Educação de pós-graduação em enfermagem.

COMO REFERENCIAR ESTE ARTIGO:

Maffissoni AL, Sanes M da S, Schneider F, Martini JG, Lino MM, Kempfer SS. Expressão das tendências pedagógicas em teses e dissertações de um laboratório de pesquisa e tecnologia em educação em enfermagem e saúde (1994-2007). Cogitare enferm. [Internet]. 2019 [acesso em "colocar data de acesso, dia, mês abreviado e ano"]; 24. Disponível em: http://dx.doi.org/10.5380/ce.v24i0.62147.

Este obra está licenciado com uma Licença Creative Commons Atribuição 4.0 Internacional.

${ }^{1}$ Enfermeiro. Mestrando em Enfermagem. Universidade Federal de Santa Catarina. Florianópolis, SC, Brasil. $\odot$ ${ }^{2}$ Enfermeira. Doutoranda em Enfermagem. Universidade Federal de Santa Catarina. Florianópolis, SC, Brasil. () ${ }^{3}$ Enfermeira. Doutoranda em Enfermagem. Universidade Federal de Santa Catarina. Florianópolis, SC, Brasil. $\bigcirc$ ${ }^{4}$ Enfermeira. Doutora em Educação. Docente de Enfermagem e Coordenadora do Programa de Pós-Graduação em Enfermagem da Universidade Federal de Santa Catarina. Florianópolis, SC, Brasil. $(0$

${ }^{5}$ Enfermeira. Doutora em Enfermagem. Docente de Enfermagem da Universidade Federal de Santa Catarina. Florianópolis, SC, Brasil.

${ }^{6}$ Enfermeira. Doutora em Enfermagem. Docente de Enfermagem da Universidade Federal de Santa Catarina. Florianópolis, SC, Brasil. 


\title{
PEDAGOGICAL TRENDS IN THESES AND DISSERTATIONS OF A RESEARCH AND TECHNOLOGY LABORATORY IN NURSING AND HEALTH EDUCATION (1994-2017)
}

\begin{abstract}
Objective: To analyze pedagogical trends present in the scientific investigations conducted by the Research and Technology Laboratory in Nursing and Health Education of the Federal University of Santa Catarina.

Method: This was a qualitative documentary analysis of theses and dissertations produced in 1994-2017. The data were collected between March and May 2018. Content analysis was used to process the data.

Results: A total of 106 documents were analyzed. Of these, 89 were affiliated with the liberating/creative trend, 17 with the progressive trend, and no studies were found that were exclusively related to the conventional pedagogical trend.

Conclusions: The theses and dissertations produced by the Research Lab addressed nursing education on a perspective mostly based on the liberation/creative pedagogical trend. This study contributed to constructing knowledge by recognizing the pedagogical assumptions used by researchers in the field, essential for identifying the political positions of Brazilian nursing when conducting research
\end{abstract}

DESCRIPTORS: Nursing research; Research groups; Educational models; Nursing education; Nursing graduate education.

\section{EXPRESIÓN DE TENDENCIAS PEDAGÓGICAS EN TESSIS Y DISERTACIONES DE LABORATORIO DE INVESTIGACIÓN Y TECNOLOGIA EDUCATIVA EN ENFERMERÍA Y SALUD (1994-2017)}

\begin{abstract}
RESUMEN:
Objetivo: Analizar la expresión de las tendencias pedagógicas en las investigaciones científicas del Laboratorio de Investigación y Tecnología en Educación en Enfermería y Salud, Universidad Federal de Santa Catarina.

Método: Investigación cualitativa, aplicando análisis documental de tesis y disertaciones producidas en el período 1994-2017. Datos recolectados entre marzo y mayo de 2018. Datos estudiados por análisis de contenido.

Resultados: Se identificaron 106 documentos. De ellos, 89 adoptan la tendencia liberadora/ creativa, 17 se acercan a la tendencia progresista, yo no hubo evidencia de estudios exclusivamente relacionados a la tendencia pedagógica convencional.

Conclusiones: Las tesis y disertaciones del Laboratorio de Investigación trabajaron la educación en enfermería a partir de una perspectiva establecida, mayoritariamente, por la tendencia liberadora/creativa. Este estudio contribuye a la construcción del conocimiento en enfermería, al reconocer los presupuestos pedagógicos utilizados por investigadores del área, esencial para identificar las posturas políticas que la enfermería brasileña asume en sus investigaciones científicas
\end{abstract}

DESCRIPTORES: Investigación en Enfermería; Grupos de Investigación; Modelos Educacionales; Educación en Enfermería; Educación de Posgrado en Enfermería. 
Entre a emergência da pesquisa nos anos 1950 e a contemporaneidade da produção do conhecimento, a enfermagem tem assumido, paulatinamente, sua prática científica e social. Nota-se uma busca constante no sentido de aproximar os saberes produzidos, a partir das necessidades sociais em saúde, e consolidar a investigação científica como ato político e recurso de fortalecimento da enfermagem no Brasil ${ }^{(1)}$.

Nesse cenário, a pós-graduação figura locus de expressiva contribuição para a produção de conhecimento em enfermagem, considerando que nos últimos 20 anos ocorreram articulações significativas entre as necessidades de saúde/doença da população e os centros de formação no âmbito acadêmico, o que torna o processo investigativo conectado à realidade brasileira.

No âmbito da pós-graduação, os grupos de pesquisa representam o espaço hegemônico de produção de conhecimentos e formação de recursos humanos em pesquisa, pois permitem aprofundamento teórico necessário ao campo de saber, impulsionando os profissionais para o desenvolvimento de postura investigativa crítica e reflexiva ${ }^{(1)}$, ao mesmo passo que são importantes dispositivos para o fortalecimento da identidade profissional na área da enfermagem ${ }^{(2)}$. Assim, a prática nestes espaços parte da premissa de uma produção científica que conecte pesquisadores de diversos níveis de formação, integrados por áreas afins, com o objetivo de compartilhar ideias e percorrer trajetórias investigativas de forma coletiva, integrada e sistematizada ${ }^{(3)}$.

Especificamente no contexto da produção de conhecimento em educação em enfermagem no Brasil, desde 1992, o Laboratório de Pesquisa e Tecnologia em Educação em Enfermagem e Saúde (EDEN) do Programa de Pós-graduação em Enfermagem da Universidade Federal de Santa Catarina (PEN/UFSC), centra suas investigações no compromisso com a qualificação do ensino e do cuidado em enfermagem e na saúde, lançando esforços para a compreensão de questões relacionadas ao ato de educar, aos processos de ensinar e aprender e aos agentes do processo educativo ${ }^{(4)}$.

Em um percurso sistematizado ao longo de seus 25 anos, o grupo desenvolve pesquisas a fim de consolidar a produção do conhecimento em educação em enfermagem, contemplando o objetivo fundamental de desenvolver investigação, ação e reflexão na área de ensino de enfermagem, em nível de graduação, pós-graduaçã̃o e educação permanente, por meio da união entre os diversos atores inseridos neste contexto e mediante utilização de metodologias e tecnologias capazes de aperfeiçoar os processos de aprendizagem ${ }^{(5)}$.

Ademais, observa-se que o constructo educação assume diversas perspectivas que se entremeiam às práticas sociais, culturais, econômicas e políticas de determinada sociedade, em dado momento da história. Assim, a educação em enfermagem acompanha este movimento, sendo influenciada pelas práticas educativas vigentes, o que desvela a coexistência, também na produção do conhecimento em enfermagem, de correntes educativas com paradigmas distintos, que estão assentadas em posturas pedagógicas, denominadas como convencionais, progressistas e/ou libertadoras ${ }^{(6)}$.

Entende-se como tendência pedagógica convencional aquele processo educativo que visa à acomodação, com a finalidade de manter a ordem social, sem modificar a sociedade, ao passo que a tendência progressista visa à mudança das pessoas para atender às necessidades sociais. Para, além disso, a tendência libertadora assume compromisso com a mudança da sociedade, no sentido de explicitar as injustiças e modificá-las.

Ao evidenciar que a produção de conhecimento em enfermagem deve dialogar com as demandas de saúde $e^{(2-7)}$, é essencial compreender como as diferentes posturas pedagógicas se expressam nas práticas investigativas da área. Além disso, há destacado interesse em priorizar estudos acerca de referenciais teórico-metodológicos de educação em saúde $e^{(8)}$, formação de profissionais para os sistemas de saúde ${ }^{(9)}$, cobertura universal(10), e metodologias educacionais ${ }^{(11)}$. 
Isto posto, objetivou-se analisar como as tendências pedagógicas se expressam nas teses e dissertações do grupo de pesquisa EDEN/UFSC, ao longo dos 25 anos de sua existência. O recorte temporal entre os anos de 1994 e de 2017 foi adotado em função de as duas datas representarem, respectivamente, a mais antiga e a mais recente produção científica, provenientes do grupo e defendidas no PEN/UFSC.

\section{MÉTODO}

Trata-se de uma pesquisa qualitativa, com delineamento descritivo e exploratório, realizada a partir de análise documental de teses e dissertações produzidas pelo EDEN/ UFSC, no período de 1994 a 2017. A análise documental pressupõe o exercício de identificar, verificar e apreciar documentos relacionados a determinado objeto, o que permite ao pesquisador conhecer e se aproximar de um processo, algo que se constituiu e se constitui ao longo do tempo na história de pessoas, grupos, ideias e/ou práticas ${ }^{(12-13)}$.

O mapeamento das teses de doutorado e dissertações de mestrado seguiu dois percursos: primeiramente, foi consultada a lista de defesas de teses e sustentação de dissertações do Programa de Pós-Graduação em Enfermagem da Universidade Federal de Santa Catarina (PPGENF-UFSC) por membros do EDEN; num segundo momento, efetuouse a recuperação destes documentos na página virtual do programa. $O$ universo de estudos compreendeu um total de 43 teses e 63 dissertações, considerando que 1994 foi o ano da primeira dissertação sustentada, 2000 o ano da primeira tese defendida e 2017 foi ano mais recente com defesa de tese e sustentação de dissertação.

A coleta das informações e análise dos dados foi realizada nos meses de março, abril e maio de 2018, mediante leitura integral das categorias, resumo, resultados, discussão e considerações finais, e da leitura dinâmica dos trabalhos completos. Os dados coletados foram inseridos em planilha no Microsoft Excel $\circledR^{\circ}$ e sistematizados quanto à natureza de pesquisa (tese ou dissertação), ano de publicação, título, doutorando(a)/ mestrando(a), orientadora, objetivos gerais, referencial teórico, abordagem de pesquisa adotada (qualitativa, quantitativa ou quanti-qualitativa), metodologia desenvolvida, temáticas centrais da produção e tendência pedagógica, que poderia ser libertadora/ criativa, progressista ou convencional. A Figura 1 traz informações mais detalhadas sobre as principais características de cada tendência pedagógica. 


\begin{tabular}{|c|c|c|c|}
\hline & LBERTADORCRLATIVO & PROCRESSISTA & CONVENCIONAL \\
\hline Funglo & Vse a trandormuge. & Vasardorma & Vise a acomodage. \\
\hline Consesjiknclas & 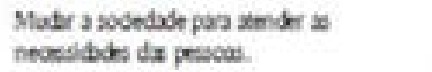 & 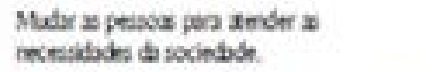 & Inpedir mudncas, manter a ondkin socal. \\
\hline Estrategla & $\begin{array}{l}\text { oporses a desipaldsde inpestipe } \\
\text { composo. }\end{array}$ & 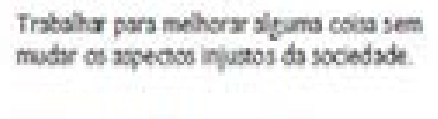 & 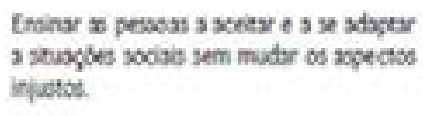 \\
\hline Intenglo & Ltwtat, oter progeas axcil. & 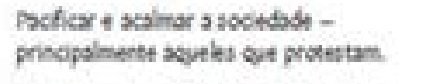 & Cortrodar a socindade. \\
\hline Enfogere & 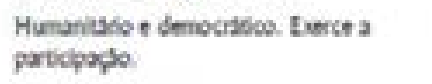 & $\begin{array}{l}\text { Putemalats. Eurct um certesin } \\
\text { "bondewo". }\end{array}$ & Autondia. Eusct un contoh rigibo. \\
\hline $\begin{array}{l}\text { Efelito na } \\
\text { coemunidade }\end{array}$ & 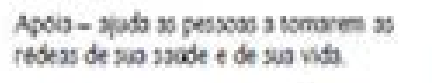 & 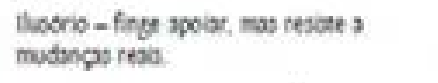 & 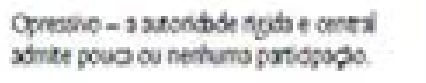 \\
\hline $\begin{array}{l}\text { Como os } \\
\text { particlpantes salo } \\
\text { perroebidos }\end{array}$ & 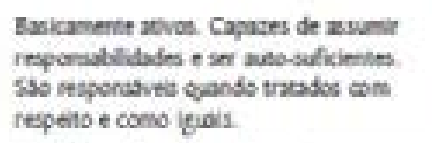 & 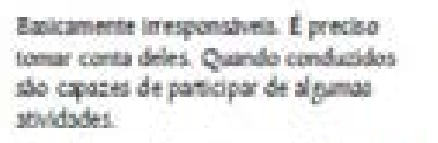 & 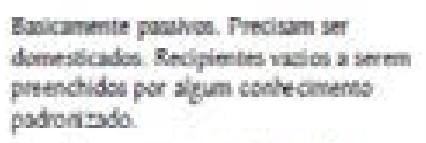 \\
\hline $\begin{array}{l}\text { Metrodo de } \\
\text { ensino }\end{array}$ & 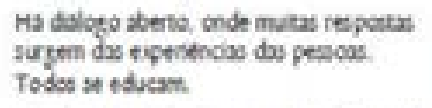 & 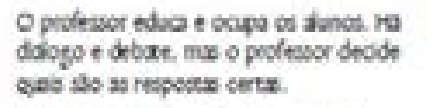 & 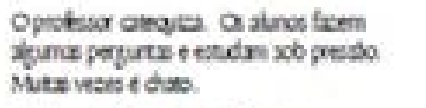 \\
\hline $\begin{array}{l}\text { Modo de } \\
\text { aprender }\end{array}$ & 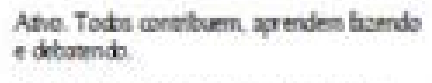 & $\begin{array}{l}\text { Mak ou menos atvo. Decorar andse } \\
\text { funtomerol. }\end{array}$ & 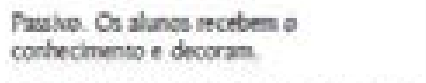 \\
\hline $\begin{array}{l}\text { Ruos de iditas e } \\
\text { coerhedimerto }\end{array}$ & MSo dupla todos sabens is teducan. & 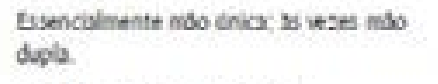 & $\begin{array}{l}\text { Nobo inicar de quen sube pars quin nada } \\
\text { sate. }\end{array}$ \\
\hline Local de ensho & A propera uda & $\begin{array}{l}\text { Sab de ala e cuers sinisyles } \\
\text { controlatal }\end{array}$ & Sala de aula. \\
\hline $\begin{array}{l}\text { Interseclo do } \\
\text { gupo }\end{array}$ & 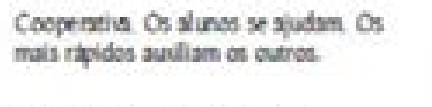 & 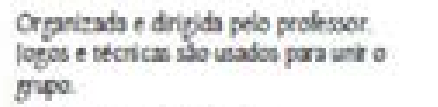 & $\begin{array}{l}\text { Compentiva Cocoproplo max provas enter } \\
\text { os allunos t dunada of frade. }\end{array}$ \\
\hline Avalbelo & 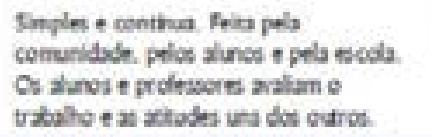 & 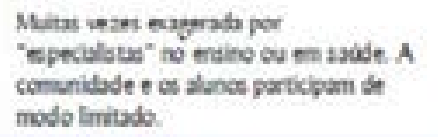 & 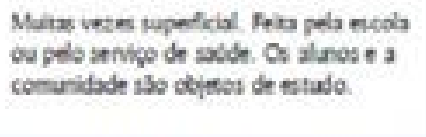 \\
\hline
\end{tabular}

Figura 1 - Concepções que orientam a prática pedagógica do processo ensino-aprendizagem. Florianópolis, SC, Brasil, 2018

Fonte: LINO et al, 2011.

No sentido de trazer completude para a expressão das tendências pedagógicas, excertos textuais foram utilizados para demonstrar as aproximações entre as ideias presentes nas pesquisas. Para a identificação dos estudos, utilizou-se T01, T02 (...) para teses e D01, D02 (...) para dissertações, seguido do ano de publicação.

A análise dos dados ocorreu por meio da técnica de análise temática de conteúdo para pesquisas qualitativas ${ }^{(14)}$. Após análise do material coletado, os dados foram agrupados em tabelas e discutidos por meio da análise qualitativa.

Por se tratar de uma pesquisa documental, este estudo não foi submetido ao Comitê de Ética em Pesquisa com Seres Humanos, entretanto, seguiram-se os preceitos éticos necessários na análise e divulgação dos dados.

\section{RESULTADOS}

Os trabalhos acadêmicos produzidos no EDEN totalizaram 106 pesquisas (43 teses e 63 dissertações), orientados por cinco pesquisadoras ao longo dos 25 anos de existência do grupo. Houve estabilidade quanto às orientadoras, o que pode ser confirmado ao observar o tempo médio de permanência no grupo, que foi de 13,2 anos. Os estudos são hegemonicamente de natureza qualitativa, mas também é possível observar o desenvolvimento de pesquisas de métodos mistos e de natureza quantitativa. 
Quanto aos objetivos das investigações, 56 pesquisas tencionaram desvelar ou caracterizar fenômenos e significações; 49 desenvolveram alguma forma de intervenção com a população pesquisada; e uma se dedicou especificamente sobre a análise reflexiva acerca de determinado assunto.

Observa-se que os estudos abordam assuntos como educação em saúde, educação em enfermagem, educação continuada, educação permanente, educação popular, currículo, formação profissional, formação docente, educação a distância, práticas e posturas pedagógicas, processos de ensino-aprendizagem, modos de produção de conhecimento, avaliação educacional e formação e desenvolvimento de grupos de pesquisa.

Com relação aos referenciais teóricos adotados, percebe-se que a maior parte das teses e dissertações utilizou teorias ou legados teóricos de autores(as) de notório saber na área da educação e/ou da enfermagem para embasar as discussões sobre as temáticas abordadas. As informações demonstraram aderência dos trabalhos a, pelo menos, 38 referenciais teóricos distintos, com maior frequência de utilização dos princípios da Pedagogia Crítico-Reflexiva, de Paulo Freire, Teoria do Conhecimento Base para o Ensino, de Lee Shulman, e Teoria da Ação Comunicativa, de Jürgen Habermas.

Ao buscar aproximação entre as posições político-pedagógicas presentes nas teses e dissertações e os pressupostos das tendências pedagógicas, nota-se que a maioria dos estudos explorou suas temáticas de interesse a partir de uma perspectiva relacionada ao ensino libertador/criativo. Outras pesquisas teceram discussões com base nos preceitos da educação progressista e não foram evidenciados trabalhos com interpretações caracterizadas exclusivamente pela tendência pedagógica convencional, conforme apresentado na Tabela 1.

Tabela 1 - Tendências Pedagógicas das Teses e Dissertações produzidas pelo Grupo EDEN-UFSC. Florianópolis, SC, Brasil, 2019

\begin{tabular}{lcccc} 
Período & \multicolumn{2}{c}{ Libertadora/Crítica } & \multicolumn{2}{c}{ Progressista } \\
\cline { 2 - 5 } & Teses & Dissertações & Teses & Dissertações \\
\hline 1994 a 1998 & - & 8 & - & 1 \\
\hline 1999 a 2003 & 2 & 13 & 1 & 5 \\
\hline 2004 a 2008 & 9 & 9 & 2 & 0 \\
\hline 2009 a 2013 & 9 & 15 & 1 & 2 \\
\hline 2014 a 2017 & 18 & 6 & 1 & 4 \\
\hline Total & 38 & 51 & 5 & 12
\end{tabular}

Fonte: os(as) autores(as), 2019.

O conteúdo de grande parte das teses e dissertações demonstrou que as posturas adotadas pelos(as) pesquisadores(as) no desenvolvimento de suas pesquisas estão imbuídas por concepções de ensino-aprendizagem como um processo dinâmico e em constante construção. O respeito à cultura e aos saberes dos sujeitos, a importância de relações pedagógicas fundamentadas no diálogo, o entendimento dos movimentos educacionais como dependentes do contexto social e da educação como estratégia de estímulo à tomada de consciência e consequente prática de liberdade, são características que permearam as pesquisas do EDEN. 
Através, então, da valorização do saber, um saber oriundo da história das comunidades e da vida dos indivíduos, as ações de educação e saúde estimulavam um situar-se no mundo, saindo do silêncio e de uma postura receptiva de modelos e prescrições. (D04-1997)

Retratamos a importância do compartilhamento de saberes por meio do diálogo, que é potencial transformador da realidade. Destacamos que, implicado no processo de conhecer, está a tarefa de intervir. Intervir para a libertação, para a transformação das condições injustas, de vida e de saúde. (T18-2011)

Os aspectos presentes nos excertos indicaram aderência das pesquisas às premissas da tendência pedagógica libertadora/criativa. A prática dialógica e a concepção dos processos educacionais foram compreendidas enquanto dispositivos transformadores da realidade.

Se a professora propicia esta nova forma de intervir, a relação pedagógica se processará de maneira diferente do habitual, pois tanto ela [professora] quanto a aluna não será mera executora/reprodutora de tarefas e normas, mas sim pensadoras críticas, que propõem novas possibilidades frente ao que está colocado. Assim, para formação de uma profissional crítico-criativa, precisamos construir tecnologias de ação do trabalho vivo em ato, e de gestão deste trabalho pedagógico, que provoquem ruídos, abrindo fissuras e linhas de fuga, nos processos de trabalho, instituído nos ambientes acadêmicos que, por si só, devem ser espaços de liberdade. (T04- 2004)

Somente ao compreender a pedagogia libertadora como um meio capaz de promover a visão ampliada de cuidado, se efetuará a mudança de Estilo de Pensamento necessária para desenvolver enfermeiros crítico- criativos hábeis para oferecer um cuidado integral ao outro e a si mesmo. (D44-2010)

Além disso, nota-se a compreensão da educação para além da transmissão de conhecimentos e saberes. Houve sinergia entre as reflexões presentes nos estudos e a tendência pedagógica libertadora/criativa, as quais se fundamentam no princípio de que o(a) professor(a), o(a) estudante, a comunidade e quem mais estiver envolvido nestes movimentos podem (e devem) acreditar na educação como ato político, vislumbrando a partir dela promover modificações capazes de superar as iniquidades da sociedade.

Para sair da obscuridade é preciso iluminar-se. Iluminar-se significa estar consciente dessa escuridão e a partir da "tomada de consciência" decidir e sair dela. É no movimento de estar no mundo, admirá-lo, refletir sobre ele e criar que o homem se torna homem. Essa característica pertence somente ao homem e somente ele pode transformar o mundo e se transformar. (T21-2012)

E assim, mesmo que esbarrem na irracional ideologia capitalista e faça com que, nos ambientes produtivos, a educação em saúde ainda tenda a se fundamentar em prescrições técnicas e comportamentais para a manutenção da saúde dos trabalhadores, acredita-se que vivências de práticas educativas críticas em saúde, com mulheres trabalhadoras, em ambientes fabris, permitam um processo de reflexão pessoal, ainda que apresentando limitações condicionadas por esse contexto de trabalho. (T30 - 2015)

Em relação ao conjunto de teses e dissertações categorizado como de maior aproximação à tendência pedagógica progressista, nota-se a compreensão dos processos educativos a partir de uma lógica de adaptação às estruturas sociais vigentes. Tais trabalhos demonstraram concepções de educação como estratégia profícua para trabalhar algumas questões da sociedade, contudo, sem alterar de forma significativa ou revolucionária as injustiças presentes nela.

No entanto, somente o tempo irá evidenciar alguma mudança concreta. O objetivo pretendido de 'não revolucionar', mas despertar para uma prática educativa considera-se, portanto, alcançado. (D13 - 2000)

Esse estudo tornou possível refletir a respeito dessas vivências e das necessidades inerentes 
ao preparo dos egressos para a inserção no mercado de trabalho e planejamento da sua carreira profissional e científica (D56 - 2014)

Nesta parcela de estudos evidencia-se também a interpretação do papel do(a) educador(a) como detentor(a) do conhecimento. Foi conferido a este agente o dever de transmitir, repassar ou disseminar os saberes que possui para instruir/formar/capacitar os indivíduos.

Tanto os egressos quanto os gerentes de enfermagem descreveram o empenho e compromisso dos professores em formarem Técnicos em Enfermagem para exercer a profissão de forma competente e segura. (T32 - 2015)

Cabe ao docente conhecer seu aluno e nivelar seu conhecimento, conhecer suas necessidades, pontos fracos a serem desenvolvidos, e dessa forma alcançar de forma prazerosa e eficaz a aprendizagem do conteúdo pelo discente. (D63 - 2017)

\section{DISCUSSÃO}

As pesquisas do EDEN/UFSC seguem a tendência de produção científica na enfermagem, considerando a inquietude com a compreensão dos significados e dos fenômenos que os indivíduos atribuem às suas experiências e práticas. A proximidade das investigações com objetos de pesquisa inseridos em temáticas atuais e que carecem de maior aprofundamento teórico-científico, pode demonstrar a responsabilidade das investigadoras em responder às necessidades sociais com a produção dos trabalhos científicos no grupo.

As temáticas que apareceram com maior frequência nas produções do EDEN são também focalizadas por outros autores ${ }^{(15)}$, quando consideram existir uma relação de dependência entre a prática educativa e a atuação do(a) enfermeiro(a) nos serviços de saúde. Assim, reconhece-se a imprescindibilidade de investigar as questões relacionadas à educação(16-17) e o compromisso assumido pelo EDEN com esse processo de investigação.

A preocupação com as questões pedagógicas na enfermagem começou a ganhar maior visibilidade no final do século $X X^{(1)}$, o que denota espaço curto de tempo para consolidação dos pressupostos da educação na produção científica em enfermagem. No entanto, já é possível, pela expressão destas tendências pedagógicas, considerar que as produções do EDEN vêm acompanhando progressivamente tais preocupações nos diferentes espaços de atenção, cuidado, ensino e gestão(18).

Nota-se a forma fluida como as tendências pedagógicas e, hegemonicamente, a tendência libertadora/criativa, se expressam ao longo do processo de escrita das teses e dissertações, considerando que em meio ao processo investigativo os autores demonstram suas filiações teóricas, ideias de mundo, concepções sobre ser humano, aprendizagem e cuidado. Exemplo disto são os referencias teóricos utilizados, com destaque para o educador Paulo Freire. Para este autor, a educação/formação é um processo de troca de saberes e experiências, pautado por movimentos dialógicos e plurais, com vistas à integração entre os temas estudados e a realidade do(a) educando(a), em busca de um itinerário educativo, sustentado pela tríade ação-reflexão-ação, que possa estimular a tomada de consciência dos(as) participantes sobre a realidade social na qual estão inseridos - uma autêntica práxis ${ }^{(19)}$.

A expressão das tendências pedagógicas na produção científica do EDEN e a manifestação mais evidente da postura libertadora/criativa parece acompanhar um movimento imperativo de mudança nas relações pedagógicas, abertas ao diálogo e centradas na aprendizagem dos(as) estudantes ${ }^{(20)}$. Essa postura rompe com a racionalidade essencialmente técnica e cria condições para a prática investigativa e educativa que se faz no encontro com o outro, a partir do respeito e da valorização dos participantes, o que, 
inclusive, tece relações com ações baseadas na perspectiva da integralidade ${ }^{(21)}$.

Considerando que estas posturas também se expressam nos modos de fazer pesquisa, observa-se nas teses e dissertações que diversas estratégias podem contribuir para a expressão de uma tendência libertadora nas investigações sobre a educação em enfermagem, como a adoção de metodologias ativas e a valorização do saber empírico. Ainda assim, existe o desafio de melhor elucidar as questões conceituais e operacionais destes movimentos, sobretudo acerca das metodologias ativas, no sentido de dar condições aos docentes na oferta de experiências educativas significativas, centradas nos estudantes, que se associem à tendência libertadora/criativa ${ }^{(22)}$.

$\mathrm{Na}$ contemporaneidade, a educação em enfermagem vivencia uma transição paradigmática, buscando atender às novas demandas profissionais, relacionadas, principalmente à necessidade de enfermeiros(as) críticos-reflexivos(as), preparados(as) para o trabalho coletivo e integrado, comprometidos com a mudança social|(21).

A adoção do enfoque crítico-reflexivo no processo de formação pode refletir no processo de trabalho em saúde e de enfermagem com potencialidades na afirmação de um cuidar realizado por sujeitos com valores, cultura e ideologia comprometidos para solução dos problemas concretos de saúde da população e dos serviços de saúde ${ }^{(23)}$.

Para que a educação em enfermagem se direcione para posturas pedagógicas libertadoras, é importante que os grupos de pesquisa da área fomentem o desenvolvimento de trabalhos que promovam o debate, demonstrem experiências pedagógicas exitosas e desvelem os resultados da formação a partir de processos pedagógicos diferenciados. Tal atividade certamente contribuirá com as (trans)formações que a enfermagem necessita frente aos novos desafios do mundo contemporâneo ${ }^{(24)}$.

Acredita-se que, mesmo com significativa expressão da tendência pedagógica libertadora/criativa, é relevante centrar esforços para a construção coletiva de estímulo às novas possibilidades neste campo. O ambiente de ensino e, em especial, o da saúde, se revela, cotidianamente, como um local de disputas e de relações de poder, no qual o processo ensino-aprendizagem pode ficar à mercê de posturas pedagógicas tradicionais (rígidas), contribuindo insuficientemente para a formação de enfermeiros(as) reflexivos(as) e transformadores(as) da realidade social (21). Neste sentido, é importante instigar movimentos capazes de estimular uma formação libertadora, que valorize o conhecimento de cada um e fomente uma atuação dos estudantes enquanto agentes de transformação dos ambientes nos quais estão inseridos.

Cumpre destacar como dificuldades para realização do estudo as dissemelhanças entre o formato organizacional dos documentos, sendo que houve maior dificuldade em desvelar informações dos estudos mais antigos; o desafio em classificar os estudos conforme as tendências pedagógicas, tendo em vista que algumas pesquisas pareciam pertencer em ambas tendências libertadora/criativa e convencional; e a pluralidade de temáticas investigadas. Ressalta-se, ainda, que o estudo se limitou em analisar documentos de um grupo de pesquisa específico, deste modo, não cabe atribuir generalizações aos resultados descritos. Por outro lado, este fato não impede que pesquisas com objetos de investigação similares possam utilizá-lo como base para desenvolver suas discussões e/ou sinalizar semelhanças e distanciamentos acerca do tema.

\section{CONSIDERAÇÕES FINAIS}

A análise documental das produções científicas permitiu a construção de novas interlocuções entre as teses e dissertações, as linhas de pesquisa e objetivos do EDEN, possibilitando recriar uma rede de sentidos entre os trabalhos, seus autores e referenciais adotados, conectados às tendências pedagógicas aqui discutidas. 
Nesse sentido, a produção de teses e dissertações do EDEN, ao longo dos seus 25 anos, brinda um percurso concebido por pesquisadoras que cultivaram o desafio de trabalhar a educação em enfermagem a partir de uma perspectiva pautada, majoritariamente, pelos pressupostos da tendência pedagógica libertadora/criativa. Destaca-se a consolidação do grupo pelo seu tempo de existência, assim como pela busca dos seus objetivos e contribuição para o cenário nacional.

Sublinha-se a importância de que práticas investigativas desenvolvidas pelos grupos de pesquisa em educação e enfermagem possam fomentar o desenvolvimento de trabalhos que promovam problematização e debate, que permitam socializar experiências pedagógicas exitosas e compartilhar os resultados da formação, a partir de processos pedagógicos diferenciados.

Agrega-se, ainda, a relevância dos estudos na esfera da educação em enfermagem como propulsores de reflexões sobre como a formação de enfermeiras e enfermeiros vem se dando, colocando em questão, de modo supremo, que a formação profissional deve estar conectada com a realidade social da população e com suas necessidades de saúde.

Finalmente, ao reconhecer que as posturas pedagógicas também embasam e estruturam o pensamento dos(as) pesquisadores(as) e a suas práticas investigativas, tornase fundamental refletir sobre a formação de pesquisadores num enfoque crítico, criativo e reflexivo (formação pedagógica do investigador).

\section{REFERÊNCIAS}

1. Padilha MICS, Borenstein M, Santos I. Enfermagem: história de uma profissão. 2. ed. São Paulo: Difusão; 2015.

2. Lino MM, Backes VMS, Costa MASMC da, Martins MMFPS, Lino MM. Pesquisa em enfermagem: Brasil e Portugal na construção da identidade profissional. Texto contexto- enferm. [Internet]. 2018 [acesso em 25 jun 2018]; 27(1). Disponível em: http://dx.doi.org/10.1590/0104-07072018006550015.

3. Erdmann AL, Peiter CC, Lanzoni GMM. Grupos de pesquisa em enfermagem no Brasil: comparação dos perfis de 2006 e 2016. Rev. Gaúcha Enferm. [Internet]. 2017 [acesso em 27 jun 2018]; 38(2).

Disponível em: http://dx.doi.org/10.1590/1983-1447.2017.02.69051.

4. Universidade Federal de Santa Catarina. Laboratório de Pesquisa e Tecnologia em Educação em Enfermagem e Saúde - EDEn: Origem do Grupo. [Internet] 2018 [acesso em 25 jun 2018]. Disponível em: http://eden.paginas.ufsc.br/origem-do-grupo/.

5. Conselho Nacional de Desenvolvimento Científico e Tecnológico (CNPQ). Espelho Grupo de pesquisa Laboratório de Pesquisa e Tecnologia em Educação em Enfermagem e Saúde - EDEN. [Internet] 2018 [acesso em 25 jun 2018]. Disponível em: http://dgp.cnpq.br/dgp/ espelhogrupo/4744110645361888\#repercussao.

6. Lino MM, Backes VMS, Ferraz F, Reibnitz KS, Martini JG. Análise da produção científica dos grupos de pesquisa em educação em enfermagem da região sul do Brasil. Texto contexto - enferm. [Internet]. 2010 [acesso em 25 jun 2018]; 19(2). Disponível em: http://dx.doi.org/10.1590/S0104-07072010000200007.

7. Costa ACB, Chaves ECL, Terra FS, Monteira LA. Perfil dos grupos de pesquisas de Enfermagem do Conselho Nacional de Desenvolvimento Científico e Tecnológico. Rev. RENE. [Internet]. 2014 [acesso em 25 jun 2018]; 15(3). Disponível: http://dx.doi.org/10.15253/2175-6783.2014000300012.

8. Brasil. Ministério da Saúde. Agenda nacional de prioridades de pesquisa em saúde. 2. ed. Brasília: Editora do Ministério da Saúde; 2015.

9. Organización Panamericana de la Salud. Formación doctoral en enfermería en América Latina y el Caribe. [Internet] Washington: OPS; 2017 [acesso em 25 mar 2019]. Disponível em: https://www. 
observatoriorh.org/formacion-doctoral-en-enfermeria-en-america-latina-y-el-caribe.

10. Cassiani SHB, Bassalobre-Garcia A, Reveiz L. Acesso Universal à Saúde e Cobertura Universal de Saúde: identificação de prioridades de pesquisa em enfermagem na América Latina. Rev. LatinoAm. Enfermagem. [Internet]. 2015 [acesso em 25 jun 2018]; 23(6). Disponível em: http://dx.doi. org/10.1590/0104-1169.1075.2667.

11. Scochi CGS, Gelbcke FL, Ferreira MA, Lima MADS, Padilha KG, Padovani NA, et al. Doutorado em Enfermagem no Brasil: formação em pesquisa e produção de teses. Rev. Latino-Am. Enfermagem. [Internet]. 2015 [acesso em 25 jun 2018]; 23(3). Disponível em: http://dx.doi.org/10.1590/01041169.0590 .2564 .

12. Padilha MI, Bellaguarda MLR, Nelson S, Maia ARC, Costa R. O uso das fontes na condução da pesquisa histórica. Texto contexto - enferm. [Internet]. 2017 [acesso em 25 jun 2018]; 26(4). Disponível em: http://dx.doi.org/10.1590/0104-07072017002760017.

13. Andrade SR de, Schmitt MD, Storck BC, Piccoli T, Ruoff AB. Análise documental nas teses de enfermagem: técnica de coleta de dados e método de pesquisa. Cogitare enferm. [Internet]. 2018 [acesso em 25 jun 2018]; 23(1). Disponível em: http://dx.doi.org/10.5380/ce.v23i1.53598.

14. Minayo MCS. O desafio do conhecimento: pesquisa qualitativa em saúde. 4. ed. São Paulo: Hucitec; 2014.

15. Püschel VAA, Costa D, Reis PP, Oliveira LB de, Carbogim FC. Nurses in the labor market: professional insertion, competencies and skills. Rev. bras. enferm. [Internet]. 2017 [acesso em 25 jun 2018]; 70(6). Disponível em: http://dx.doi.org/10.1590/0034-7167-2016-0061.

16. Chrizostimo MM, Brandão AAP. A formação profissional do enfermeiro: 'estado da arte'. Enferm glob [Internet]. 2015 [acesso em 25 jun 2018]; 14(4). Disponível em: http://dx.doi.org/10.6018/ eglobal.14.4.208841.

17. Abrahao AL, Merhy EE. Formação em saúde e micropolítica: sobre conceitos-ferramentas na prática de ensinar. Interface. [Internet]. 2014 [acesso em 25 jun 2018]; 18(49). Disponível em: http://dx.doi. org/10.1590/1807-57622013.0166.

18. Rodrigues CCFM, Carvalho DPSRP, Salvador PTCO, Medeiros SM, Menezes RMP, Ferreira Júnior MA, et al. Ensino inovador de enfermagem a partir da perspectiva das epistemologias do Sul. Esc. Anna Nery. [Internet]. 2016 [acesso em 25 jun 2018]; 20(2). Disponível em: http://dx.doi.org/10.5935/14148145.20160053.

19. Mendonça ET de, Cotta RMM, Lelis VP, Carvalho Junior PM. Paradigms and trends in higher education: the action research methodology as a teacher education strategy. Interface. [Internet]. 2015 [acesso em 25 jun 2018]; 19(53). Disponível em: http://dx.doi.org/10.1590/1807-57622013.1024.

20. Conterno SFR, Lopes RE. Pressupostos pedagógicos das atuais propostas de formação superior em saúde no Brasil: origens históricas e fundamentos teóricos. Avaliação. [Internet]. 2016 [acesso em 25 jun 2018]; 21(3). Disponível em: http://dx.doi.org/10.1590/S1414-40772016000300016.

21. Lima MM de, Reibnitz KS, Kloh D, Silva KL da, Ferraz F. Relação pedagógica no ensino práticoreflexivo: elementos característicos do ensino da integralidade na formação do enfermeiro. Texto contexto - enferm. [Internet]. 2018 [acesso em 25 jun 2018]; 27(2). Disponível em: http://dx.doi. org/10.1590/0104-070720180001810016.

22. Meira MDD, Kurcgant P. Nursing education: training evaluation by graduates, employers and teachers. Rev. bras. enferm. [Internet]. 2016 [acesso em 25 jun 2018]; 69(1). Disponível em: http://dx.doi. org/10.1590/0034-7167.2016690102i.

23. Silva LAA da, Soder RM, Oliveski CC, Frizzo MN. Program for Improvement and Revitalization of Knowledge in Nursing and Health. Interface. [Internet]. 2018 [acesso em 25 jun 2018]; 22(65). Disponível em: http://dx.doi.org/10.1590/1807-57622016.0629. 
24. Canever BP, Prado ML do, Backes VMS, Schveitzer MC. Tendências pedagógicas na produção do conhecimento em educação em enfermagem do estado de São Paulo. Rev. bras. enferm. [Internet]. 2013 [acesso em 25 jun 2018]; 66(6). Disponível em: http://dx.doi.org/10.1590/S0034-71672013000600019.

Recebido: 06/10/2018

Finalizado: 06/05/2019

Autor Correspondente:

André Lucas Maffissoni

Universidade Federal de Santa Catarina

R. Eng. Agronômico Andrei Cristian Ferreira, s/n - 88040-900 - Florianópolis, SC, Brasil

E-mail: andremaffissoni@hotmail.com

\section{Contribuição dos autores:}

Contribuições substanciais para a concepção ou desenho do estudo; ou a aquisição, análise ou interpretação de dados do estudo - ALM, MSS, FS

Elaboração e revisão crítica do conteúdo intelectual do estudo - MML, SSK

Aprovação da versão final do estudo a ser publicado - MML, SSK

Responsável por todos os aspectos do estudo, assegurando as questões de precisão ou integridade de qualquer parte do estudo - JGM 